\title{
Creation of Matter in a Noncommutative Universe
}

\author{
Tomasz Miller \\ Faculty of Mathematics and Information Science, \\ Warsaw University of Technology \\ ul. Koszykowa 75, 00-662 Warsaw, Poland \\ and Copernicus Center for Interdisciplinary Studies, \\ Cracow, Poland \\ Michael Heller \\ Copernicus Center for Interdisciplinary Studies, Cracow, Poland \\ and Vatican Observatory, V-00120 Vatican City State
}

March 25, 2021

\begin{abstract}
The dark matter and dark energy problem, that is now dominating the research in cosmology, makes the question of the origin of mass-energy content of the universe more urgent than ever. There are two philosophies regarding this question: according to Mach's principle it is matter that generates geometry of space-time, and according to Wheeler's geometrodynamics some configurations of space-time geometry are to be interpreted as its material content. Neither of these philosophies has led to success. In the present paper, we show that there exists an algebraic generalisation of geometry that reconciles, in a sense, these two seemingly opposite standpoints. The geometry is constructed with the help of a noncommutative algebra of smooth functions on a groupoid and its derivations. The groupoid in question has a nice physical interpretation: it can be regarded as a space of Lorentz rotations. In this way, Lorentz symmetries are inherent
\end{abstract}


to the generalised geometry of space-time. We define the action for this geometry and, by varying it, obtain generalised vacuum Einstein equations (for a simplified model). It turns out that these equations contain additional terms (with respect to the standard vacuum Einstein equations) which are naturally interpreted as the components of the energy-momentum tensor. Matter is thus created out of purely geometric degrees of freedom. We find two exact solutions (for even more simplified case). We argue that the creation of matter, being a global effect, makes the contrast between Mach and Wheeler philosophies ineffective.

\section{Introduction}

The dark matter and dark energy problem, that is now dominating the research in cosmology, makes the question of the origin of mass-energy content of the universe more urgent than ever. This problem could hardly be solved without a deeper understanding of matter creation mechanisms in the universe. Einstein's equations are plagued with a dualism of geometry (left hand side of the equations) and matter (their right hand side). Einstein looked for a remedy against this difficulty in the idea called by him Mach's principle. It admits several nonequivalent formulations, some stronger of which claim that (local) geometry should totally be determined by the global distribution of matter throughout space-time. The idea could be encapsulated in the slogan "geometry out of matter" 3]. The opposite philosophy was propagated by John Archibald Wheeler who argued that the material sources that appear in Einstein's equations can entirely be reconstructed from a characteristic imprint they exert on the space-time geometry. Here we have a slogan "matter out of geometry". The program was known as "Wheeler's geometrodynamics" [19, 20, 21]. The fact that neither of these philosophies, attractive as they are, has led to the success suggests that a stumbling block lies in the very concept of geometry that is too rigid to accommodate for such a complex phenomenon as that of matter. In the present paper, we explore a generalisation of the standard differential geometry and, basing on it, construct cosmological models in which two above mentioned philosophies not only seem to work, but also could, in a sense, be unified.

There are two ways of doing differential geometry: by using local coordinate systems, and algebraically in terms of smooth functions on a given space, the latter being independent of the choice of coordinates. Both these 
methods are equivalent, but the second is better suited for generalisations. Let us then consider a space-time manifold $M$ and the algebra $C^{\infty}(M)$ of smooth functions on $M$. The algebra $C^{\infty}(M)$ can, without losing its geometric properties, be replaced by a more general, not necessarily commutative, algebra on a more general space than the space-time manifold $M$. The more general space we use to this end is the so-called groupoid, denoted by $\Gamma$ (for details see Section 2). The choice of this space has a nice physical motivation. the groupoid concept is a generalisation of the group concept, and as such it generalizes the notion of symmetry. In our case, the elements of the groupoid $\Gamma$ can be regarded as Lorentz rotations. Therefore, $\Gamma$ can serve as a more natural environment for general relativity (being the space of its symmetries) than the usual "naked" space-time.

We then consider the algebra $C^{\infty}(\Gamma)$ of smooth (compactly supported) functions on a groupoid $\Gamma$. The consequence of these rather simple replacements is that space-time points acquire their internal structure, i.e. internal degrees of freedom not unlike in the Kaluza-Klein type models, with the difference that now internal degrees of freedom are really "internal", not created by adding new space dimensions.

The algebra $C^{\infty}(\Gamma)$ can easily be made into a $C^{*}$-algebra which assimilates the formalism to that employed in quantum mechanics. In fact, this approach has been used to construct a model unifying general relativity and quantum mechanics with a perspective to make an attempt at a quantum gravity theory [8, 9, 10, 11]. It goes without saying that truly fundamental mechanism of matter creation can hardly be imagined without the correctly working quantum gravity theory. This is why the present work can only be regarded as a preliminary step in this direction.

Models presented in this paper are nothing more than "toy models"; however, they seem worthwhile to be explored not only because they give such surprising results as far as matter generation is concerned, but also because they are preparing mathematical tools to deal with more realistic situations. The present work is based on our research paper [12].

The plan of our essay runs as follows. In Section 2, we briefly discuss the method of doing geometry on the groupoid $\Gamma$ in terms of the algebra $C^{\infty}(\Gamma)$ and its derivations. In Section 3, we construct such a geometry for a groupoid with a finite structure group. Then, in Section 4, we deduce generalised Einstein equations from the corresponding action principle, and show that they, when projected onto space-time, contain an additional term that can naturally be interpreted as a "matter source". We also find two 
explicit "Friedman-like" solutions for a simplified form of the metric. Finally, we append some concluding remarks.

\section{Space of Lorentz Symmetries and Its Ge- ometry}

A natural setting for relativity theory is a set of pairs of reference frames with a Lorentz transformation acting between them. This setting can be given strict mathematical form. Let $M$ be a space-time, and let us consider the bundle of reference frames (frame bundle, for short) on $M$, denoted by $\left(E, \pi_{M}\right)$ where $E$, called the total space of the bundle, is the set of all local reference frames on $M$, and $\pi_{M}: E \rightarrow M$ is a mapping projecting a frame $p \in E$ to its attachment point $x \in M, \pi_{M}(p)=x$. The set of all frames attached to $x, E_{x}=\pi_{m}^{-1}(x)$, is called the fiber at $x$. Let $G$ be a Lorentz group or one of its subgroups. It acts on $E$ along fibres, i.e. any two frames in the same fibre can be transformed into each other with the help of an element of $G 1$ In this way, the Cartesian product has been constructed,

$$
\Gamma=E \times G=\{\gamma=(p, g): p \in E, g \in G\} .
$$

It is clear that Lorentz transformations in a given fibre can, as elements of a group, be suitably composed and have inverses. The construction, just described, is called transformation groupoid with $\Gamma$ as its groupoid space, $E$ its base space, and $G$ its structure group. This purely algebraic construction can be equipped with the smoothness structure; it is then called smooth transformation groupoid.

Just as geometry of space-time $M$ can be done in terms of the algebra $C^{\infty}(M)$ of smooth functions on $M$, a generalised geometry of space-time $M$ can be done in terms of an algebra $\mathcal{A}$ on the groupoid $\Gamma=E \times G$. The usual algebra $C^{\infty}(\Gamma)$ of smooth (compactly supported) functions on $\Gamma$

(with pointwise multiplication, denoted by ·) would reproduce geometry of $\Gamma$ with nothing interesting for our program. To obtain an interesting generalisation we replace the commutative pointwise multiplication with a not necessarily commutative multiplication $*$. Its concrete form need not bother us here (for details see [12]). We thus consider the algebra $\mathcal{A}=\left(C^{\infty}(\Gamma), *\right)$

\footnotetext{
${ }^{1}$ This means that we consider only Lorentz rotations; translations, i.e. transformations between fibres, are excluded.
} 
of smooth (compactly supported) functions on $\Gamma$. It is, in general, noncommutative which means that if $a, b \in \mathcal{A}$ then $a * b \neq b * a$. This seemingly innocuous modification leads to dramatic changes in geometry. We also impose on $\mathcal{A}$ suitable smoothness conditions.

Which is the contact of the algebra $\mathcal{A}$ with the algebra $C^{\infty}(M)$ of smooth functions on space-time $M$ ? To answer this question we remind the concept of the center of a noncommutative algebra. The center of an algebra $A$, denoted $\mathcal{Z}(A)$ is the set of all elements of $A$ that commute with all elements of $A$ (it is obvious that if an algebra is commutative, it coincides with its center). It can be shown that the center of our algebra $\mathcal{A}$ is isomorphic with $C^{\infty}(M)$. Therefore, if, in constructing geometry, we restrict the algebra $\mathcal{A}$ to its center $\mathcal{Z}(\mathcal{A})$, we obtain the geometry of space-time $M$.

The standard manifold geometry is encoded in its metric tensor. Mathematically, metric is a function taking two smooth vector fields as its arguments and returning a smooth function on this manifold. In the algebraic approach to geometry, the counterparts of vector fields are derivations of the corresponding algebra. Let us consider our algebra $\mathcal{A}$. Its derivation is a linear map $v: \mathcal{A} \rightarrow \mathcal{A}$ satisfying the well known Leibniz rule

$$
v(a * b)=v(a) * b+a * v(b)
$$

for $a, b \in \mathcal{A}$. The set of all derivations of the algebra $\mathcal{A}$ is denoted by $\operatorname{Der}(\mathcal{A})$.

Taking the above into account, we assume the metric of the form $\mathcal{G}$ : $V \times V \rightarrow \mathcal{Z}(\mathcal{A})$ where $V \subseteq \operatorname{Der}(\mathcal{A}) 2$ The pair $(\mathcal{A}, V)$ is called differential algebra. It serves us as the basic structure to construct an algebraic version of generalised geometry. We proceed in strict analogy to what is usually done when developing the standard differential geometry. We construct connection (with the help of the Koszul formula), curvature and all other magnitudes necessary to write down Einstein's field equations. In the following, when pursuing this program, we shall limit ourselves to a special case of a finite model.

\footnotetext{
${ }^{2} V$ should have the $\mathcal{Z}(\mathcal{A})$-module structure as well as the Lie algebra structure.
} 


\section{Geometry on the Groupoid Algebra with Finite Structure Group}

Let us consider an $n$-element subgroup $G$ of the group of Lorentz rotations. Without loss of generality, we can assume that the frame bundle studied is trivial: $E=M \times G$, and therefore the considered groupoid is

$$
\Gamma=M \times G \times G=\left\{\left(x, g_{i}, g_{j}\right): x \in M, g_{i}, g_{j} \in G, i, j=1, \ldots, n\right\} .
$$

The noncommutative multiplication $*$ in the algebra $C^{\infty}(\Gamma)$ of smooth (compactly supported) functions on $\Gamma$ is given by

$$
\forall a, b \in C^{\infty}(\Gamma) \quad(a * b)\left(x, g_{k}, g_{l}\right):=\sum_{m=1}^{n} a\left(x, g_{k}, g_{m}\right) b\left(x, g_{k} g_{m}, g_{m}^{-1} g_{l}\right) .
$$

It is much more convenient, however, to regard every function $a \in C^{\infty}(\Gamma)$ as an $n$-by-n matrix $\left(a_{i j}\right)$ with $C^{\infty}(M)$-valued entries, defined via

$$
a_{i j}(x):=a\left(x, g_{i}, g_{i}^{-1} g_{j}\right) .
$$

In this way, the algebra studied becomes

$$
\mathcal{A}_{n}:=\mathbb{M}_{n}\left(C^{\infty}(M)\right)=C^{\infty}(M) \otimes \mathbb{M}_{n}(\mathbb{C})
$$

and the operation $*$ becomes nothing but the standard matrix multiplication

$$
\forall a, b \in \mathcal{A}_{n} \quad(a * b)_{i j}(x):=\sum_{k=1}^{n} a_{i k}(x) b_{k j}(x) .
$$

Let us note that algebras similar to $\mathcal{A}_{n}$ commonly appear in the exploration of possible applications of noncommutative geometry to physics, most notably in the context of the Noncommutative Standard Model of particle physics (see [6, 4] for details on noncommutative geometry and the Noncommutative Standard Model or [7, 14] for a more accessible review).

The center $\mathcal{Z}\left(\mathcal{A}_{n}\right)$ of this algebra consists of matrices of the form $f I_{n}$, where $f \in C^{\infty}(M)$ and $I_{n}$ denotes the $n$-by- $n$ identity matrix. It is, therefore, isomorphic to the algebra $C^{\infty}(M)$, just as the previous section anticipated. From now on we shall identify $\mathcal{Z}\left(\mathcal{A}_{n}\right)$ with $C^{\infty}(M)$.

We consider the full $C^{\infty}(M)$-module of derivations of the algebra $\mathcal{A}_{n}$, $V:=\operatorname{Der} \mathcal{A}_{n}$. It can be shown that $V$ decomposes into the direct sum of two its submodules: 
- The submodule Hor $\mathcal{A}_{n}$ of horizontal derivations. Its elements are liftings of the smooth vector fields on the manifold $M$ onto $\mathcal{A}_{n}$. More explicitly, for any smooth vector field $X \in \operatorname{Der} C^{\infty}(M)$ one defines its lifting as a map $\bar{X}: \mathcal{A}_{n} \rightarrow \mathcal{A}_{n}$ acting entrywise, namely

$$
\forall a \in \mathcal{A}_{n} \quad(\bar{X} a)_{i j}:=X a_{i j} .
$$

- The submodule Inn $\mathcal{A}_{n}$ of inner derivations. By an inner derivation induced by an element $b \in \mathcal{A}_{n}$ one understands a map $\operatorname{ad}_{b}: \mathcal{A}_{n} \rightarrow \mathcal{A}_{n}$ defined as

$$
\forall a \in \mathcal{A}_{n} \quad \operatorname{ad}_{b} a:=[b, a]=b * a-a * b .
$$

Embarking on the construction of the generalised geometry on the differential algebra $\left(\mathcal{A}_{n}, V\right)$, one begins with the metric $\mathcal{G}: V \times V \rightarrow \mathcal{Z}(\mathcal{A})$, upon which the Levi-Civita connection and the curvature tensors are subsequently defined. All these objects can be, and usually are, studied by physicists with the help of the so-called abstract-index notation. The idea behind this notation is to identify the abstract (usually tensorial) objects with the array of their components in a chosen basis of $V$. Multi-indexed expressions, obtained in this way, can then be effectively manipulated under the set of simple rules, among others including the Einstein summation convention (see [18, Chapter 2]).

The natural choice for the (local) basis of Hor $\mathcal{A}_{n}$ is the lifting of the $c 0$ ordinate basis $\left(\frac{\bar{\partial}}{\partial x^{\mu}}\right)$ induced by some chart $x$. In the following, we shall denote the liftings of the coordinate vector fields $\frac{\partial}{\partial x^{\mu}}$ simply by $\partial_{\mu}$, suppressing both the overline and the reference to the inducing chart and using also other lowercase Greek letters $\nu, \lambda, \sigma, \ldots$. We adopt the usual convention that these indices can assume values $0,1, \ldots, m-1$, where $m=\operatorname{dim} M$.

On the other hand, one can show that any basis of $\operatorname{Inn} \mathcal{A}_{n}$ contains exactly $n^{2}-1$ elements. In what follows, we shall not specify the basis concretely, but we shall denote its elements by $\partial_{\{A\}}$ (by analogy with the horizontal derivations), using also other capital Latin letters in curly brackets $\{B\},\{C\},\{D\}, \ldots$. These indices can assume values $\{1\},\{2\}, \ldots,\left\{n^{2}-1\right\}$ (interpreted as the "inner degrees of freedom"). The use of curly brackets assures that we do not mix the values of indices of the two types.

Summarizing, the basis of $V$ contains two kinds of elements: the horizontal derivations and the inner derivations, and this is reflected in the two types 
of indices used: the lowercase Greek indices and the capital Latin indices in curly brackets. Additionally, it will be convenient to write $\partial_{A}$ for a generic derivation from the basis, using also other capital Latin letters $B, C, D, \ldots$ These indices can take all values assumed by the indices of the two types listed above.

Let us note here that the Einstein summation convention applies to each of the three types of indices separately.

The noncommutativity of $\mathcal{A}_{n}$ has a direct effect on the commutation relations between $\partial_{A}$ 's. Even though $\left[\partial_{\mu}, \partial_{\nu}\right]=0$, exactly as for the coordinate basis in the standard differential geometry, in general we have that $\left[\partial_{A}, \partial_{B}\right] \neq 0$. Mathematically speaking, some of the structure constants $\mathbf{c}_{A B}{ }^{C}$, defined by the formula $\left[\partial_{A}, \partial_{B}\right]=\mathbf{c}_{A B}{ }^{C} \partial_{C}$, are nonzero.

Having specified the basis, we can now construct the generalised geometry on the differential algebra $\left(\mathcal{A}_{n}, V\right)$. In the abstract-index notation, the metric $\mathcal{G}$ is represented by the doubly indexed array $g_{A B}$ where

$$
g_{A B}:=\mathcal{G}\left(\partial_{A}, \partial_{B}\right)
$$

One can regard $g_{A B}$ as a square, symmetric and nonsingular matrix of order $m+n^{2}-1$. Its inverse matrix is denoted by $g^{A B}$. Exactly as in the standard differential geometry, the metric matrix and its inverse can be used to lower and raise indices of other multi-indexed entities.

We are now ready to define the Levi-Civita connection $\nabla: V \times V \rightarrow V$ by means of the Koszul formula. Namely, for any $u, v \in V, \nabla_{u} v$ is the unique derivation which satisfies

$$
\begin{aligned}
\mathcal{G}\left(\nabla_{u} v, w\right): & =\frac{1}{2}[u(\mathcal{G}(v, w))+v(\mathcal{G}(u, w))-w(\mathcal{G}(u, v)) \\
& +\mathcal{G}(w,[u, v])+\mathcal{G}(v,[w, u])-\mathcal{G}(u,[v, w])] .
\end{aligned}
$$

for all $w \in V$. The connection's components (called the Christoffel symbols of the second kind) are defined by the equality

$$
\nabla_{\partial_{C}} \partial_{B}=\Gamma_{B C}^{A} \partial_{A}
$$

and by the Koszul formula they can be expressed as

$$
\Gamma_{B C}^{A}=\frac{1}{2} g^{A D}\left(\partial_{C} g_{D B}+\partial_{B} g_{D C}-\partial_{D} g_{B C}+\mathbf{c}_{C B D}+\mathbf{c}_{D C B}-\mathbf{c}_{B D C}\right) .
$$


Due to the noncommutativity, we have

$$
\Gamma_{B C}^{A}-\Gamma_{C B}^{A}=\mathbf{c}_{C B}{ }^{A} .
$$

Therefore, unlike the components of the standard Levi-Civita connection, $\Gamma_{B C}^{A}$ might not be symmetric with respect to the latter two indices.

In spite of this asymmetry, the Levi-Civita connection enjoys many of the properties of its standard counterpart, in particular, it is torsion-free, that is

$$
\forall u, v \in V \quad \nabla_{u} v-\nabla_{v} u-[u, v]=0
$$

and compatible with the metric, that is

$$
\forall u, v, w \in V \quad w(\mathcal{G}(u, v))=\mathcal{G}\left(\nabla_{w} u, v\right)+\mathcal{G}\left(u, \nabla_{w} v\right)
$$

Moreover, just like in the standard case, the Levi-Civita connection is the unique map $V \times V \rightarrow V$ satisfying the above two conditions.

The Riemann curvature tensor $R: V \times V \times V \rightarrow V,(u, v, w) \mapsto R(u, v) w$ is defined by means of $\nabla$ via

$$
R(u, v) w:=\nabla_{u} \nabla_{v} w-\nabla_{v} \nabla_{u} w-\nabla_{[u, v]} w .
$$

Its components $R_{D A B}^{C}$ are defined through

$$
R\left(\partial_{A}, \partial_{B}\right) \partial_{D}=R_{D A B}^{C} \partial_{C},
$$

and are given by the formula

$$
R_{D A B}^{C}=\partial_{A} \Gamma_{D B}^{C}-\partial_{B} \Gamma_{D A}^{C}+\Gamma_{D B}^{K} \Gamma_{K A}^{C}-\Gamma_{D A}^{K} \Gamma_{K B}^{C}-\mathbf{c}_{A B}{ }^{K} \Gamma_{D K}^{C} .
$$

This is nothing but the standard expression for the components of the Riemann tensor with an additional term that can be regarded as due to noncommutativity. It can be easily checked that $R$ enjoys the usual Riemann tensor symmetries

$$
\begin{aligned}
& R_{C D A B}=-R_{D C A B}=-R_{C D B A}=R_{A B C D}, \\
& R_{D A B}^{C}+R_{B D A}^{C}+R_{A B D}^{C}=0 .
\end{aligned}
$$

Finally, the Ricci tensor, ric : $V \times V \rightarrow C^{\infty}(M)$ and the curvature scalar $r \in C^{\infty}(M)$ can be introduced as suitable contractions of the Riemann tensor. Concretely, the components of the Ricci tensor read

$$
\operatorname{ric}_{A B}:=R_{A C B}^{C}
$$


whereas the curvature scalar is

$$
r:=g^{A B} \text { ric }_{A B}=g^{A B} R_{A C B}^{C} .
$$

Notice that, just as in the standard case, $\mathbf{r i c}_{A B}=\mathbf{r i c} \mathbf{c}_{B A}$.

With the generalised curvature tensors defined, we are ready to formulate and study the generalised vacuum Eistein equations.

\section{Generalised Einstein Equations}

The standard derivation of the Einstein equations is conducted by means of the action principle, starting from a suitably chosen action functional. In standard GR, the so-called Einstein-Hilbert action is the integral of the curvature scalar over the entire space-time manifold. Therefore, the natural candidate for the generalised Einstein-Hilbert action is

$$
S_{E H}:=\int r \sqrt{|g|} d^{m} x
$$

where $m=\operatorname{dim} M$ and $g$ denotes the determinant of the metric matrix $g_{A B}$. We want to study the vacuum equations and therefore postulate no additional matter term.

The action principle amounts here to varying $S_{E H}$ with respect to $\delta g^{A B}$ and assuming that the variation vanishes. The calculation leads to the generalised Einstein equations of the form

$$
\operatorname{ric}_{A B}=0 \text {. }
$$

Although these equations look similar to the standard vacuum Einstein equations, they actually have a far richer content when projected onto spacetime $M$. This is due to the extra terms coming from additional components of the metric (the "inner degrees of freedom"). These extra terms can be interpreted as an "m-dimensional matter-energy" induced by the generalised (vacuum) Einstein equations (similarly as it is the case in the Kaluza-Kleintype theories [13, 2, 17, 5], although without introducing extra geometrical dimensions). Let us consider an example of the following block diagonal metric

$$
g_{A B}=\left[\begin{array}{cc}
g_{\mu \nu} & 0 \\
0 & g_{\{A\}\{B\}}
\end{array}\right] .
$$


Let us remember that $g_{\mu \nu}$ is an $m$-by- $m$ matrix and $g_{\{A\}\{B\}}$ is a $\left(n^{2}-1\right)$-by$\left(n^{2}-1\right)$ matrix, where $n=|G|$. The inverse metric matrix $g^{A B}$ is also block diagonal.

Such block diagonal metric has an important feature: its corresponding Levi-Civita connection $\nabla$ extends the standard Levi-Civita connection $\widetilde{\nabla}$ : $\operatorname{Der} C^{\infty}(M) \times \operatorname{Der} C^{\infty}(M) \rightarrow \operatorname{Der} C^{\infty}(M)$ in the sense that

$$
\forall \bar{X}, \bar{Y} \in \operatorname{Hor} \mathcal{A}_{n} \quad \nabla_{\bar{X}} \bar{Y}=\overline{\widetilde{\nabla}_{X} Y} .
$$

In other words, the Levi-Civita connection acts on horizontal derivations in the same way as does its classical counterpart.

For the block diagonal metric, the generalised Einstein equations can be written more explicitly than (2) as

$$
\begin{aligned}
& \widetilde{\mathbf{r i c}}_{\mu \nu}=\frac{1}{4}\left(\widetilde{\nabla}_{\mu} \widetilde{\nabla}_{\nu} \ln |\breve{g}|+g^{\{A\}\{B\}} \widetilde{\nabla}_{\mu} \widetilde{\nabla}_{\nu} g_{\{A\}\{B\}}\right), \\
& \mathbf{c}_{\{B\}}\{C\}\{D\} \\
& \widetilde{\Delta} \partial_{\{C\}\{D\}}=0, \\
& \quad=-2 \mathbf{c}_{\{A\}}\{C\}\{D\} \\
& \quad\left(\mathbf{c}_{\{B\}\{C\}\{D\}}+\mathbf{c}_{\{B\}\{D\}\{C\}}\right)+\mathbf{c}_{\{C\}\{D\}}^{\{A\}} \mathbf{c}_{\{C\}\{D\}\{B\}} .
\end{aligned}
$$

where

- $\widetilde{\text { ric }}_{\mu \nu}$ denotes the standard Ricci tensor.

- $\widetilde{\nabla}_{\mu}$ is the covariant derivative resulting from the standard Levi-Civita connection $\widetilde{\nabla}$. Note that $\widetilde{\nabla}_{\mu}$ by definition "sees" only the lowercase Greek indices.

- $\widetilde{\Delta}:=g^{\mu \nu} \widetilde{\nabla}_{\mu} \widetilde{\nabla}_{\nu}$ is the standard Laplace-Beltrami operator.

- $\breve{g}:=\operatorname{det} g_{\{A\}\{B\}}$.

Equation (3) is a projection of the generalised Einstein equations (2) onto the $m$-dimensional space-time $M$. Since it implies that

$$
\widetilde{r}=\frac{1}{4}\left(\widetilde{\Delta} \ln |\breve{g}|+g^{\{A\}\{B\}} \widetilde{\Delta} g_{\{A\}\{B\}}\right),
$$

where $\widetilde{r}$ denotes the standard curvature scalar, therefore we can equivalently write (3) in the form of the standard Einstein equations with a certain 
nonzero energy-momentum tensor

$$
\begin{aligned}
\widetilde{G}_{\mu \nu}=\frac{1}{4} & {\left[\left(\widetilde{\nabla}_{\mu} \widetilde{\nabla}_{\nu}-\frac{1}{2} g_{\mu \nu} \widetilde{\Delta}\right) \ln |\breve{g}|\right.} \\
& \left.+g^{\{A\}\{B\}}\left(\widetilde{\nabla}_{\mu} \widetilde{\nabla}_{\nu}-\frac{1}{2} g_{\mu \nu} \widetilde{\Delta}\right) g_{\{A\}\{B\}}\right],
\end{aligned}
$$

where $\widetilde{G}_{\mu \nu}:=\widetilde{\mathbf{r i c}}_{\mu \nu}-\frac{1}{2} g_{\mu \nu} \widetilde{r}$ is the standard Einstein tensor.

The fact that there appears a nonzero energy-momentum tensor can be regarded as a realisation of the "matter out of geometry" mechanism [11] or, in this case more precisely, of the "scalar fields out of noncommutative geometry" mechanism. One can thus regard equations (4, 5) as the equations of state of those "emergent" scalar fields.

Additionally, we can also rewrite the generalised Einstein-Hilbert action (1) more explicitly. Namely

$$
S_{E H}=\int\left[\sqrt{|\breve{g}|} \widetilde{r}-\frac{1}{4} \sqrt{|\breve{g}|}\left(\widetilde{\Delta} \ln |\breve{g}|+g^{\{A\}\{B\}} \widetilde{\Delta} g_{\{A\}\{B\}}+\mathbf{C}\right)\right] \sqrt{-\tilde{g}} d^{m} x,
$$

where

- $\tilde{g}:=\operatorname{det} g_{\mu \nu}$.

- $\mathbf{C}:=\mathbf{c}^{\{B\}\{C\}\{D\}}\left(2 \mathbf{c}_{\{B\}\{D\}\{C\}}+\mathbf{c}_{\{B\}\{C\}\{D\}}\right)$.

Notice that noncommutativity enters into the action both through the additional compoments of the metric (i.e. the "inner derivation block" $g_{\{A\}\{B\}}$ ) as well as through the nonzero structure constants.

One can regard the theory, obtained in this way, as an example of a scalartensor theory 3 , which involves no less than $n^{2}\left(n^{2}-1\right) / 2$ independent scalar fields. These fields, when arranged into a symmetrical matrix $g_{\{A\}\{B\}}$, are such that $\breve{g}:=\operatorname{det} g_{\{A\}\{B\}}$ is a nowhere vanishing field. Notice, moreover, that $\sqrt{|\breve{g}|}$ seems to play a special role in this theory, as it can be shown to satisfy the following Klein-Gordon-like equation

$$
\left(\widetilde{\Delta}+\frac{1}{4} \mathbf{C}\right) \sqrt{|\breve{g}|}=0
$$

with $\frac{1}{4} \mathbf{C}$ playing the role of the mass term. This might constitute another illustration of how noncommutative geometry gives rise to (massive) scalar

\footnotetext{
${ }^{3}$ See e.g. [5] and references therein.
} 
fields in this model.

We now move to presenting two explicit solutions of Einstein equations (375) in the simplest case when the structure group $G$ has only two elements. We restrict ourselves to "Friedman-like" solutions, by which we mean metrics, whose "horizontal derivation block" $g_{\mu \nu}$ is of the Friedman-LemaitreRobertson-Walker form and whose remaining components are time-dependent only

footnoteAs usual, we set $c=G=1$.

$$
\begin{aligned}
& g_{\mu \nu}=\left[\begin{array}{cccc}
-1 & 0 & 0 & 0 \\
0 & \frac{a^{2}(t)}{1-k r^{2}} & 0 & 0 \\
0 & 0 & a^{2}(t) r^{2} & 0 \\
0 & 0 & 0 & a^{2}(t) r^{2} \sin ^{2} \theta
\end{array}\right], \\
& g_{\{A\}\{B\}}=g_{\{A\}\{B\}}(t) .
\end{aligned}
$$

Recall that $a(t)$ is called the scale factor and $k \in\{-1,0,1\}$ is the curvature constant.

To simplify the calculations, we assume furthermore that the "inner derivation block" $g_{\{A\}\{B\}}$ has the following form

$$
g_{\{A\}\{B\}}(t)=\left[\begin{array}{ccc}
\xi f^{2}(t) & 0 & 0 \\
0 & 0 & \eta f^{2}(t) \\
0 & \eta f^{2}(t) & 0
\end{array}\right],
$$

where $\xi, \eta \in \mathbb{R} \backslash\{0\}$ and $f$ is a time-dependent nowhere vanishing function.

One of the Einstein equations, (4), is satisfied automatically. The remaining two equations (3, 5) yield the following overdetermined nonlinear system of ordinary differential equations

$$
\left\{\begin{aligned}
\frac{\ddot{a}}{a}+\frac{\ddot{f}}{f} & =0, \\
a \ddot{a} f+2 \dot{a}^{2} f+3 a \dot{a} \dot{f} & =-2 k f, \\
f \ddot{f} a+2 \dot{f}^{2} a+3 f \dot{f} \dot{a} & =\frac{1}{\eta} a .
\end{aligned}\right.
$$

together with an additional algebraical condition that $\xi=2 \eta$.

Notice that, by the last of the above equations the function $f$ cannot be constant. Moreover, the Hubble parameter $H$ can be expressed entirely in terms of $f$ and $\eta$ via

$$
H:=\frac{\dot{a}}{a}=\frac{\eta^{-1}-f \ddot{f}-2 \dot{f}^{2}}{3 f \dot{f}} .
$$


Two explicit solutions of system (9), one for $k=0$ and another for $k=-1$, read:

$$
\begin{array}{lll}
\text { for } k=0 & a(t)=a_{0}, & f(t)=\frac{1}{\sqrt{2 \eta}}\left(t-t_{0}\right), \\
\text { for } k=-1 & a(t)=\sqrt{\frac{2}{5}}\left(t-t_{0}\right), & f(t)=\frac{1}{\sqrt{5 \eta}}\left(t-t_{0}\right),
\end{array}
$$

where $\eta, a_{0}, t_{0}$ are constants. In fact, without any loss of generality one can take $\eta=1$ and $t_{0}=0$. Note that $g_{A B}$ becomes degenerate at $t=0$.

First solution (10) describes the flat Minkowski space-time, although it is not a static solution since $\dot{f} \neq 0$ (and the above mentioned degeneracy occurs at $t=0)$.

Second solution (11) describes a hyperbolic, linearly expanding universe with the initial singularity at $t=0$, resembling (but different from) the metric studied first by Milne [15, in which $k=-1$ but $a(t)=t$. Milne's unconventional cosmology has recently gained a renewed interest in the work of Benoit-Lévy and Chardin [1] in the form of the so-called Dirac-Milne universe, which is argued to be a viable alternative to the $\Lambda$ CMD model. What is noteworthy, both the Milne model and the model governed by (11) are free from the cosmic age problem and from the horizon problem, where the latter is solved without introducing inflation [1].

However, there is a significant difference between Milne's metric and solution (11). Namely, Milne's universe is devoid of energy-matter; it is a vacuum solution. On the other hand, solution (111) is associated to a nonzero energymatter tensor. Since the solution is Friedman-like, the energy-matter tensor describes some sort of a perfect fluid. Let us therefore see, what kind of perfect fluid is in this case induced by the (noncommutative) geometry.

The standard Einstein tensor assumes here the following (mixed) form

$$
\widetilde{G}^{\mu}{ }_{\nu}=\frac{3}{2 t^{2}} \operatorname{diag}(3,1,1,1)
$$

Therefore, by Einstein equations, the induced perfect fluid energy density $\rho$ and pressure $p$ are

$$
\rho(t)=-\frac{36 \pi}{t^{2}}, \quad p(t)=\frac{12 \pi}{t^{2}} .
$$

\footnotetext{
${ }^{4}$ There might be more, however finding them (or proving that other solutions do not exist) seems like a daunting task!
} 
Note that $\rho$ is negative and therefore in this case no classical fluid matches the induced one. In fact, such fluid would violate various energy conditions of general relativity.

Nevertheless, Friedman cosmology involving negative $\rho$ has been studied in [16], where it is argued that some realisations and extensions of quantum field theories do allow for such exotic energy forms, albeit only locally. In the article cited, the perfect fluid with $\rho<0$ and $w=p / \rho=-1 / 3$ is referred to as negative cosmic strings. The interplay between such negative forms of energy and the classical positive ones leads to interesting cosmological scenarios, also studied in [16].

\section{Concluding remarks}

How do the models constructed in the present work inscribe into philosophies (alluded to in the Introduction) concerning the relationship between matter and space-time geometry? It is straightforward that they nicely fall into the "matter out of geometry" heading. Original Wheeler's program could not succeed since the standard space-time geometry had not enough degrees of freedom to accommodate his postulates. As our work has demonstrated, noncommutative generalisation of geometry creates such possibilities. The remarkable fact is that not only the energy-momentum tensor is recovered from purely geometric degrees of freedom (without postulating additional space dimensions), but also suitable equations of state can be obtained in this way. The fact that these astonishing properties are produced in the framework of very simplified models allows us to expect even more interesting effects when more realistic models are constructed.

The main feature of noncommutative generalisation of geometry consists of a strong shift in the interplay between local and global geometric properties. In strongly noncommutative spaces, local properties are entirely engulfed by their global structure. Such spaces are nonlocal entities, in the sense that local notions in them are, in general, devoid of meaning and can only be recovered as some "limiting cases". Our algebra $\mathcal{A}$ of smooth compactly supported functions on the groupoid $\Gamma$ of Lorentz symmetries with noncommutative multiplication determines a noncommutative space with milder properties. Local properties are virtually present in it as encoded in the fact that the center $\mathcal{Z}(\mathcal{A})$ of the algebra $\mathcal{A}$ is nontrivial. Owing to this fact, the usual geometry of space-time, with all its local properties, can naturally 
be recovered.

And here we have a contact with Mach's principle. As we remember, according to its strong version all local properties of space-time should be entirely determined by space-time global structure. This is exactly what happens in our models. On the level of the algebra $\mathcal{A}$, the global structure is dominating the scene, and it is this structure that determines, through the center of $\mathcal{A}$, all local properties of space-time $M$. We could say that the noncommutative space as determined by the algebra $\mathcal{A}$ is fully Machian, and all anti-Machian properties of space-time $M$ emerge when "noncommutative symmetries" are broken to the usual space-time symmetries.

In this way, "ugly dichotomy" of space-time and matter is removed, and Einstein's starving for a monistic vision of the universe could be satisfied, albeit on the level of simplified models.

\section{References}

[1] A. Benoit-Lévy, G. Chardin, Introducing the Dirac-Milne universe, Astron. Astrophys. 537, A78 (2012).

[2] T. Appelquist, A. Chodos, P.G.O. Freund (eds),Modern Kaluza-Klein theories, Addison-Wesley, Menlo Park. 1987.

[3] J. Barbour, H. Pfister (eds), Mach's Principle. From Newton's Bucket to Quantum Gravity, Birkhäuser, Boston - Basel - Berlin, 1995.

[4] A.H. Chamseddine, A. Connes, M. Marcolli, Gravity and the standard model with neutrino mixing, Adv.Theor.Math.Phys.11:991-1089, 2007.

[5] T. Clifton, P.G. Ferreira, A. Padilla, C. Skordis, Modified Gravity and Cosmology, Phys. Rep. 513(1), 1-189 (2012).

[6] A. Connes, Noncommutative Geometry, Academic Press, New York, 1994.

[7] A. Connes, Noncommutative geometry and physics, http://www . alainconnes .org/docs/einsymp.pdf (2006).

[8] M. Heller, W. Sasin, D. Lambert, Groupoid Approach to Noncommutative Quantization of Gravity, J. Math. Phys. 38, 1997, 5840-5853. 
[9] M. Heller, W. Sasin, Noncommutative Unification of General Relativity and Quantum Mechanics, Int. J. Theor. Phys. 38, 1999, 1619-1642.

[10] M. Heller, L. Pysiak, W. Sasin, Noncommutative Dynamics of Random Operators, Int. J. Theor. Phys. 44, 2005, nr 6, 619-628.

[11] M. Heller, L. Pysiak and W. Sasin, Conceptual Unification of Gravity and Quanta, Int. J. Math. Phys. 46(10), 2494-2512 (2007).

[12] M. Heller, T. Miller, L. Pysiak and W. Sasin, Geometry and General Relativity in the Groupoid Model with a Finite Structure Group, Canadian J. Phys. 93(1), 2015, 43-54.

[13] H.C. Lee (ed), An introduction to Kaluza-Klein theories, proc. Chalk River workshop on Kaluza-Klein theories, World Scientific, Singapore. 1984.

[14] J.-H. Jureit, T. Krajewski, T. Schucker, C. A. Stephan, On the noncommutative standard model, Acta Phys.Polon.B 38:3181-3202, 2007.

[15] A.E. Milne, World-Structure and the Expansion of the Universe, Z. Astrophys. 6, 1-95 (1933).

[16] R.J. Nemiroff, R. Joshi, B.R. Patla, An Exposition on Friedmann Cosmology with Negative Energy Densities, J. Cosmol. Astropart. Phys. 2015, 06006.

[17] J.M. Overduin, and P.S. Wesson, Kaluza-Klein Gravity, Phys. Rep. 283(5-6), 303-378 (1997).

[18] R. Penrose, and W. Rindler, Spinors and Space-Time, vol. 1, Cambridge University Press, Cambridge, 1984.

[19] J.A. Wheeler, "Geometrodynamics and the Issue of the Final State", in: Relativity, Groups and Topology, C. DeWitt, B. DeWitt (eds), Gordon and Breach, 1964, pp. 315-520.

[20] J.A. Wheeler, "Superspace and the Nature of Quantum Geometrodynamics", in: Battelle Rencontres, C. DeWitt, J. A. Wheeler (eds), Benjamin, 1968, pp. 242-307. 
[21] J.A. Wheeler, "From Relativity to Mutability", in: The Physicist's Conception of Nature, J. Mehra (ed.), D. Reidel, 1973, pp. 202-247. 